\title{
Some Experimental Results in Implementing a Logic Language with Multi-head Clauses on a Transputer Architecture
}

\author{
Anna Ciampolini, Evelina Lamma, Paola Mello, Cesare Stefanelli \\ Dipartimento di Elettronica, Informatica e Sistemistica \\ Università di Bologna, Viale Risorgimento 2 \\ 40136 Bologna - Italy \\ \{anna,evelina,paola,cesare\}@deis33.cineca.it
}

\begin{abstract}
We focus on the distributed implementation of an extended logic language (called ROSE) based on multi-head clauses which can be useful to implement parallel logic objects where state change is performed by unification and recursion. Distinguishing features of ROSE are OR-parallelism with committed-choice behaviour and the ANDparallel execution of subgoals which do not share variables. We present the real implementation of ROSE on a Transputer-based architecture, and discuss some experimental results obtained by considering different allocation policies for processes. These allocation policies correspond to forms of inter- and intra-object parallelism when objects with state are implemented as ROSE programs. Obtained figures help in understanding which is the optimal allocation policy to adopt on the basis of an estimation of the time necessary for performing the distributed unification and the guard and body evaluation.
\end{abstract}

\section{Introduction}

In recent years, many efforts have been devoted to define and implement parallel logic languages. At the state of the art, however, most of the promising and widely-known implementations $[19,12,10]$ are obtained on shared-memory machines since the basic model adopted in parallel logic programming is that of STREAM-parallel languages. In STREAM-parallel languages shared variables among parallel logic processes provide a basic communication mechanism, and constraints on the unification mechanism have been introduced to rule the synchronisation of processes. However, shared variables introduce centralisation points in the resulting computational model. In the context of the Japanese project for Fifth Generation Computer Systems, focusing on the implementation of concurrent logic languages, the sharing of variables among parallel processes constitutes the main problem to be faced when aiming at realising distributed implementations [18,21]. 
The aim of the paper is to address the problem of the distributed implementation of an extended logic language based on multi-head clauses, by presenting a real implementation on a Transputer-based architecture and some experimental results.

The language we consider, called ROSE [3], is not STREAM-based: AND-parallel processes do not share variables and inter-process communication is performed via multi-head clauses. Thus, the computational model adopted in ROSE generalises existing proposals, in logic programming, based on message-passing primitives [16] and on multi-head clauses $[11,17]$.

The formal definition of ROSE and its operational semantics is given in [3] according to the true concurrent model. The main part of this paper concerns ROSE implementation.

In [1] and [9] multi-head clauses are used to implement logic objects where state change is simulated by unification and recursion. We face this issue by presenting an example and discussing how the parallel features of ROSE can be used to introduce parallelism in an Actor-like style [2].

Differently from the case of STREAM-parallel languages, the lack of shared variables between AND processes makes ROSE very suitable for a distributed implementation. This has been obtained (see [4,5]) by extending the abstract machine for Prolog (Warren Abstract Machine, WAM [22]) with new instructions and data structures supporting the creation and the communication of processes and control of non-determinism. The underlying architecture adopted for the real implementation is a parallel Multiple Instruction Multiple Data (MIMD) machine with distributed memory based on the Transputer technology [13], and the parallel model is based on message-passing.

We refer to this implementation (up to now limited to the propositional case) in order to discuss some experimental results. In the case of a distributed implementation, experimental results are highly dependent on the allocation policies adopted. We consider four different allocation policies which allow an increasing degree of distribution for the processes and thus an increasing degree of real parallelism in the computation. When ROSE clauses are interpreted as objects with state, this amounts to introducing different degrees of inter- and intra-parallelism for classes and instances. Of course, an increasing degree of distribution does not necessarily correspond to an increasing degree of performance. In fact, distributing processes in different nodes implies an higher load due both to the dynamic creation of remote processes, and also to the increasing number of messages through the network. Thus, it is convenient to distribute processes only when the overhead due to the remote creation and communication is balanced by the gain obtained in parallelising computations.

We identify three basic computations: one for the head unification (which is a multi-head unification in our case), one for the guard, and one for the body evaluation. These computations correspond in an object-oriented view, to the request acceptance (multi-head unification and guard evaluation) and the method execution (body evaluation).

The ultimate goal of our experimental results is also to show which is the optimal allocation policy to adopt for a ROSE program on the basis of an estimation of the time necessary for performing the multi-head unification (by simulating the non-propositional case) and the body evaluation.

The paper is organised as follows. In section 2 , we briefly introduce the ROSE language, its object-oriented interpretation and its process model. In section 3, we 
present the distributed implementation and the allocation policies adopted. In section 4 , we discuss the experimental results. Conclusions and future works follow.

\section{ROSE and its Process Model}

\subsection{ROSE}

In this section, we briefly overview the main features of ROSE. We concentrate ourselves on the concurrent aspects of the language while omitting the description of the sequential part. The complete definition of ROSE is reported in [3] along with its semantics and several programming examples. ROSE is a proper extension of Horn Clause Logic with committed-choice behaviour. A ROSE program consists of a finite set of guarded multi-head clauses of the form:

(i) $\mathrm{H}_{1}+\ldots+\mathrm{H}_{\mathrm{m}} \leftarrow \mathrm{G} \mid \mathrm{B}_{1}+\ldots+\mathrm{B}_{\mathrm{n}}$

The guard $\mathrm{G}$ consists of a set of built-in predicates. The " + " parallel composition operator may occur both in the left- and in the right-hand side of a clause. A multihead clause, like (i), applies to a parallel composition of atoms, $A_{1}+. .+A_{m}$ say, if there exists a substitution $\vartheta$ such that: $\vartheta=m g u\left(\left(H_{1}+\ldots+H_{m}\right),\left(A_{1}+\ldots+A_{m}\right)\right)$. For example, the clause $a(X)+b(Y) \leftarrow$ true $\mid c(X)+d(Y)$ applies to the goal $\leftarrow a(1)+a(2)+b(3)$ in two different ways. The multi-head $a(X)+b(Y)$ unifies both with $(\mathrm{a}(1)+\mathrm{b}(3))$ and with $(\mathrm{a}(2)+\mathrm{b}(3))$, and the goal may reduce either to $\leftarrow \mathrm{a}(2)+\mathrm{c}(1)+\mathrm{d}(3)$ or to $\leftarrow a(1)+c(2)+d(3)$, respectively.

The intended meaning of the guard $G$ is that a clause like (i) is applicable (candidate) to a goal if both the head unification and the guard evaluation succeed. For example, the clause:

$$
a(X)+b(Y) \leftarrow X>Y \mid c(X)+d(Y)
$$

is applicable to the goal $\leftarrow a(3)+b(1)$, but not to the goal $\leftarrow a(1)+b(1)$.

The formal definition of the operational semantics of ROSE is given in [3] according to the true concurrent model.

In ROSE restricted-AND-parallelism is supported since AND-parallel goals cannot share variables (see [3]). A current goal is maintained as multiset of atomic formulae which are possibly reduced through the applications of program clauses. ORparallelism is achieved by simultaneous applications of clauses to disjoint sub-parts of the current goal.

\subsection{Example: ROSE for Object-Oriented Programming}

Also in [1] and [9] multi-head clauses are used to implement logic objects where state change is simulated by unification and recursion.

The ROSE language can be used to implement parallel objects with state without using side-effects. A changeable object state, in fact, can be regarded as a causal chain of events. Instance variables are represented by logical variables and do not change their state by using assignment statements as in traditional object-oriented systems, but by each recursively calling itself with different state values. Thus instances must be active objects (or actors [2]) that execute continually, and suspend when there is no request. 
We can represent instances with state by using ROSE clauses. Let us consider the following ROSE program, representing the counter class:

counter(C,IName)+up(IName) $\leftarrow$ true I plus(C,1,NC), counter(NC,IName).

counter(C,IName)+show (IName,C) $\leftarrow$ true I counter(C,IName).

counter(C,IName)+reset(IName) $\leftarrow$ true I counter $(0$, IName $)$.

It specifies the behaviour of a counter as follows: an instance of counter with name IName and state $\mathrm{C}$ can accept a message up and become a counter with the new state NC (notice the comma for specifying the sequential execution of the two AND goals); it can accept a message show and maintain its old state or can accept a message reset and set its state to 0 .

Thus the first head of each clause corresponds to the instance state, while the second head corresponds to the name of the "method" to be served.

The acceptance of a method invocation involves both the multi-head unification and the guard evaluation. If the acceptance is successfully performed, the method is executed by evaluating the body of the clause. The following ROSE goal:

$$
\leftarrow \text { counter }(0, \mathrm{c} 1) \text {, up }(\mathrm{c} 1)
$$

creates a counter instance with name $c l$ and initial state 0 and then sends it a message up.

It is worth noticing that since the ROSE language is intrinsically parallel and sequentiality must be explicitly stated using the commit operator and the sequential AND, object-oriented systems based on the ROSE language present a degree of parallelism notably higher then that exploited in traditional object-oriented systems based on procedural languages and in the actor-based ones.

In fact, not only instances and methods of the same object can be evaluated in parallel (realising inter-object and intra-object parallelism respectively), but also the acceptance of requests invoking the same method, and the execution of the method can be performed in parallel (see section 4).

Notice that a similar approach has been already followed [14] starting from a STREAM-based language of the Concurrent Prolog family.

Let us consider the following Concurrent Prolog counter object:

counter([up|RemMessages],C) $\leftarrow$ truelplus $(\mathrm{C}, 1, \mathrm{NewC})$, counter(RemMessages?,NewC) counter([show $(\mathrm{C}) \mid$ RemMessages], $\mathrm{C}) \leftarrow$ true $\mid$ counter(RemMessages?,C)

counter([reset|RemMessages], C)\& true I counter(RemMessages?,0)

Notice that in this case, each instance is not identified by a name, but by its private input message stream.

A counter instance with initial state 0 is created by invoking the following goal:

$\leftarrow$ counter(S1?,0), send(S1,up,S2)...

where S1 is specified as a read-only variable to suspend the counter instance if no request is present in the stream. To send the request up to the counter the shared variable S1 must be bound to a term of the form [uplS2]. S2 is the remaining stream used, if needed, to send other messages to the same instance. The send predicate is defined as follows:

send([Msg|RemOfStream],Msg, RemOfStream).

Notice that in extensions based on Concurrent Prolog, shared logical variables are the communication means (streams) to which instances access in a single pipelined way. 
Two instances can send messages to the same object by merging their streams into the one consumed by the object. This is not the case in ROSE objects, thanks to the mechanism of multi-head clauses adopted for the communication. Thus objects based on ROSE language are more suitable for a distributed implementation. This is the subject of the following sections.

Inheritance between objects in our framework can be obtained in a way very similar to the one presented in [1]. The distinguishing feature of our work is that we address a parallel implementation of these logical objects in a distributed environment, exploiting different forms of parallelism between objects.

\subsection{The Process Model}

The ROSE computational model can be described in terms of AND/OR processes which execute asynchronously and communicate each other via message-passing primitives (see also [5]).

The AND/OR process tree of parallel logic programming [7] is replaced by a direct acyclic AND/OR graph. An initial goal of the form $\leftarrow A_{1}+\ldots+A_{k}$ is mapped onto an AND node with $k$ children $O R$ nodes, one for each atomic formula $A_{i}$ in the goal. Each OR node is labelled by some atom $A_{i}$ and has a child AND node for each clause such that at least one atom of its head unifies with $A_{i}$. A process is assigned to each node of the AND/OR graph. Following [7], each AND node corresponds to an OR process, and each OR node to an AND process.

Let us consider the clause: $\quad a(X)+b(Y) \leftarrow$ true $I c(X)+d(Y)$

The execution of the goal: $\quad \leftarrow a(1)+b(3)+a(2)$ generates the following AND/OR graph of processes (see fig. 1).

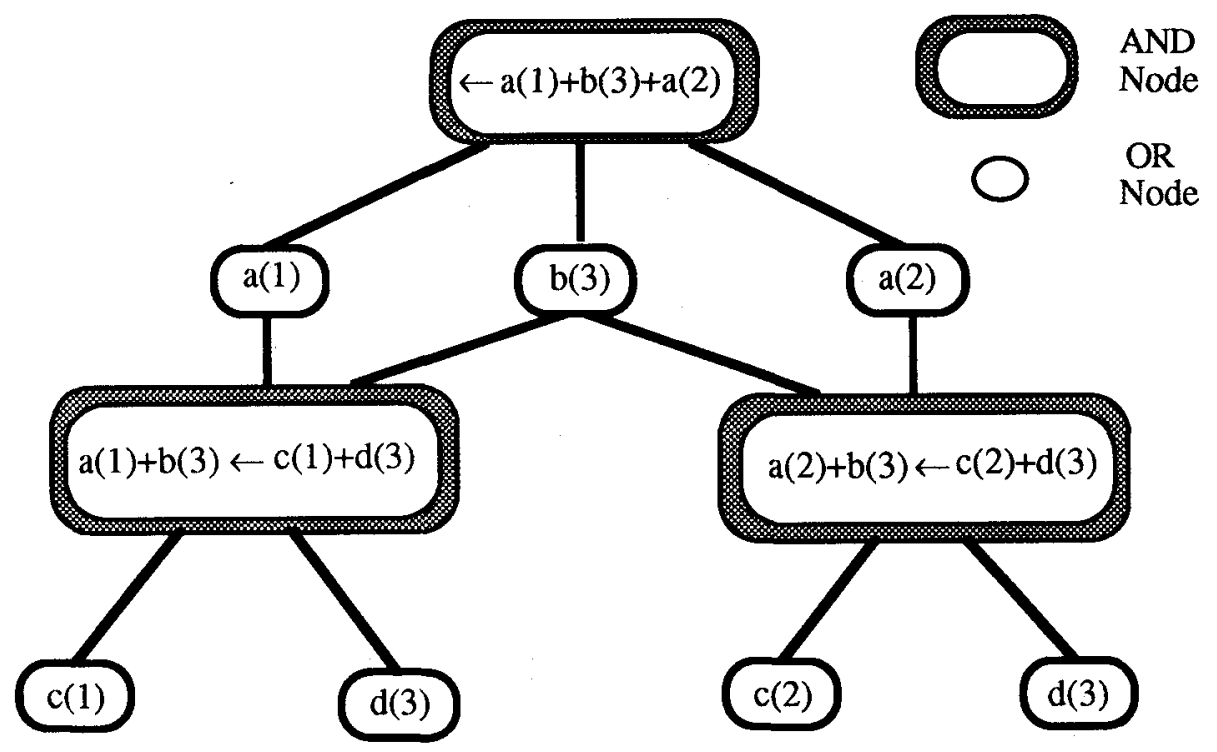

Fig. 1. AND/OR Graph. 
As in all "committed-choice" languages [19], OR-parallelism is limited to head unification and guard evaluation. The commitment to a given $O R$ process reduces the AND/OR graph. Actually, only the "committing" OR process will continue the computation, and generate the corresponding AND processes, while sibling OR processes are killed.

The process model dynamically creates several communicating processes, corresponding to every clause of the program. For each clause, a manager process is created to perform multi-head unification. Each AND process, responsible for an atomic goal $\mathrm{A}$, sends a message (A) to the manager process of a clause $C=A_{1}+\ldots+A_{n} \leftarrow$ GlBody such that at least one $A_{j}$ has the same predicate symbol of $A$. After receiving the message, the manager of $C$ creates a new child process to perform the unification of $A$ with $A_{j}$, and routes the received message to all the other children. In more details, a top-level AND parallel goal is spawned in a number of AND processes and one manager process is created for each multi-head clause. After the creation, each AND process sends a message containing its corresponding goal to the manager process of interest and then suspends, waiting for a success or a failure message. The clause manager process creates a new process to perform the head unification and forwards each unification message to its already created son processes. They, in turn, can create new children to perform other unifications at the reception of new messages. Therefore, multi-head unification, for each clause, is implemented by a tree of processes (the unification tree) where each node is represented by a unification process, and each leaf is an OR process (see fig. 2).

Processes which complete the multi-head unification phase, and therefore correspond to the OR processes of the AND/OR graph, start the guard evaluation and possibly commit.

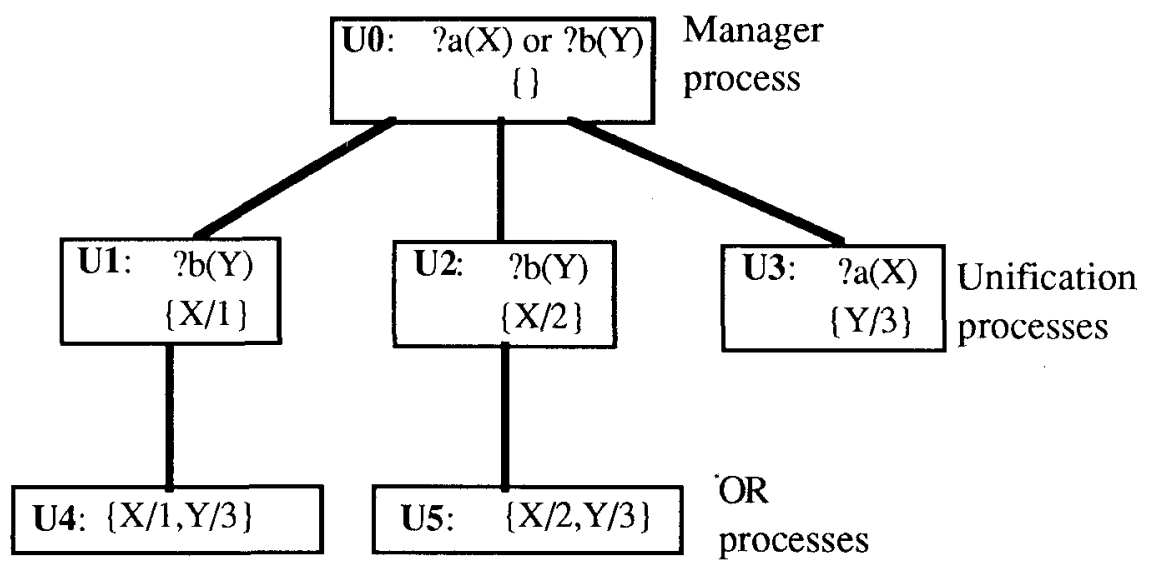

Fig. 2. The multi-head unification tree for a clause: $a(x)+b(y) \leftarrow$ truel. and the goal: $\leftarrow a(1)+a(2)+b(3)$.

The clause manager process could represent a centralisation point in a real implementation. In fact, in the model presented, all messages directed to some unification processes must deal with the clause manager. The process model described 
has to be implemented carefully, in order to avoid the transformation of the clause manager in a bottleneck for the system.

\section{The Implementation}

This section discusses the real implementation of ROSE on a parallel MIMD machine with distributed memory. Since we are mainly interested in comparing different experimental results depending on the allocation policy adopted, in this work we will omit the description of the ROSE abstract machine which can be found in [5].

Moreover, the implementation we used for experimental results takes into account only propositional programs. The case of programs containing variables and how to perform the distributed unification is discussed in [8].

\subsection{The Architecture}

We refer to a Meiko Computing Surface architecture, which is based on the Transputer technology [13]. The configuration adopted for the first implementation of ROSE comprises sixteen Transputers, each one with 4 Mbyte of local memory. With reference to the programming tools and languages to be used, the OCCAM language [6] fits well into the Transputer architecture, but it is too limited since it allows neither the dynamic creation of processes nor asynchronous communication. In the first ROSE implementation we have preferred to use CSTools [15], a more flexible programming tool obtained by extending the $\mathrm{C}$ language with primitives for process creation and interaction. The CSTools library offers a set of primitives that reduce constraints on the communication between processes, and overcomes the OCCAM channel concept by associating asymmetric, bi-directional (synchronous or asynchronous) ports with processes. Therefore, the translation of the communication protocols between processes of our distributed implementation scheme into CSTools primitives has been quite natural.

The creation of a new process on a remote node implies the presence of an active process on the remote node whose function is to create processes locally. This is due to the lack of programming tools for the dynamic remote creation of processes.

\subsection{Exploiting Parallelism}

In order to explain better the experimented allocation policies, we first point out the parallelism that can be exploited in ROSE programs.

The time spent for the complete execution of a clause $C$ :

$$
\mathrm{H}_{1}+\ldots+\mathrm{H}_{\mathrm{m}} \leftarrow \mathrm{G} \mid \mathrm{B}_{1}+\ldots+\mathrm{B}_{n}
$$

can be split into the time $T_{u}$ necessary for the multi-head unification, the time $T_{g}$ for the guard evaluation, and the time $\mathrm{T}_{\mathrm{b}}$ for the body execution $\left(\mathrm{TB}_{1}+\ldots+\mathrm{TB}_{\mathrm{n}}\right)$. From an object-oriented point of view, $\mathrm{Tu}+\mathrm{Tg}$ represents the method acceptance time, while $\mathrm{Tb}$ represents the execution time of a method.

Theoretically, we have (restricted) AND-parallelism and OR-parallelism limited to the (multi-head) unification and guard evaluation. 
Moreover, OR-parallelism makes possible to perform different clause applications in parallel. Notice that, for maximal exploitation of the parallelism, different applications of the same clause $\mathrm{C}$ (activations of $\mathrm{C}$ ) should also run in parallel.

In the first prototype of ROSE we have chosen to map all AND processes generated by an OR process, $P$, on the same physical node of $P$. In fact, the computational load of an OR process after the creation of its AND children is very light since it only waits for a result deriving from its descendants.

Where to allocate the newly created process (i.e. unification and $O R$ processes), in accordance with the allocation policies described in the following section, is determined by a static policy, not the best but the less expensive in terms of computational load. For discussion of alternative policies see [4].

\subsection{Parallelism for Objects: Allocation Policies}

Several allocation strategies are investigated here for the efficient mapping of objects onto the transputer network.

In particular, we compare different allocation policies which can be adopted for a ROSE program on the basis of an estimation of the time necessary to perform the distributed unification and the body evaluation (see section 5). For this purpose, we have considered four different allocation policies which allow an increasing degree of distribution for processes and thus an increasing degree of real parallelism for obtained objects.

1 - Single node allocation: inter-class parallelism

In this case, all the processes (the top goal, the clause managers, unification processes) are allocated on a single node. The execution of a ROSE program is therefore obtained by interleaving of the (multi-head) unification, guard evaluation and body execution of the single clause activations.

From an object-oriented point of view this policy concentrates instances of the same class in a single node. Concurrency between objects of different classes is the only form of true parallelism allowed, provided that the code of different classes be allocated on different nodes.

\section{2 - Clause managers distribution: an intra class parallelism}

In this case, the manager processes are distributed on different nodes (see figure 3 ). All the unification processes (OR processes included) belonging to the unification tree of manager $\mathrm{M}$ are allocated on the same node of $\mathrm{M}$. The manager processes being distributed onto different nodes, the execution of different clauses is truly concurrent, whereas we still have interleaving for the activations of the same clause.

In the object-oriented interpretation of ROSE, this policy allows the parallel execution of different methods, implementing a form of intra-class parallelism. Moreover, two methods can be evaluated in true parallelism (at least for the acceptance phase) even by the same instance. In this case we obtain an intra-object form of parallelism. No true parallelism for different activations of the same method (i.e., both acceptance and execution) is allowed. 


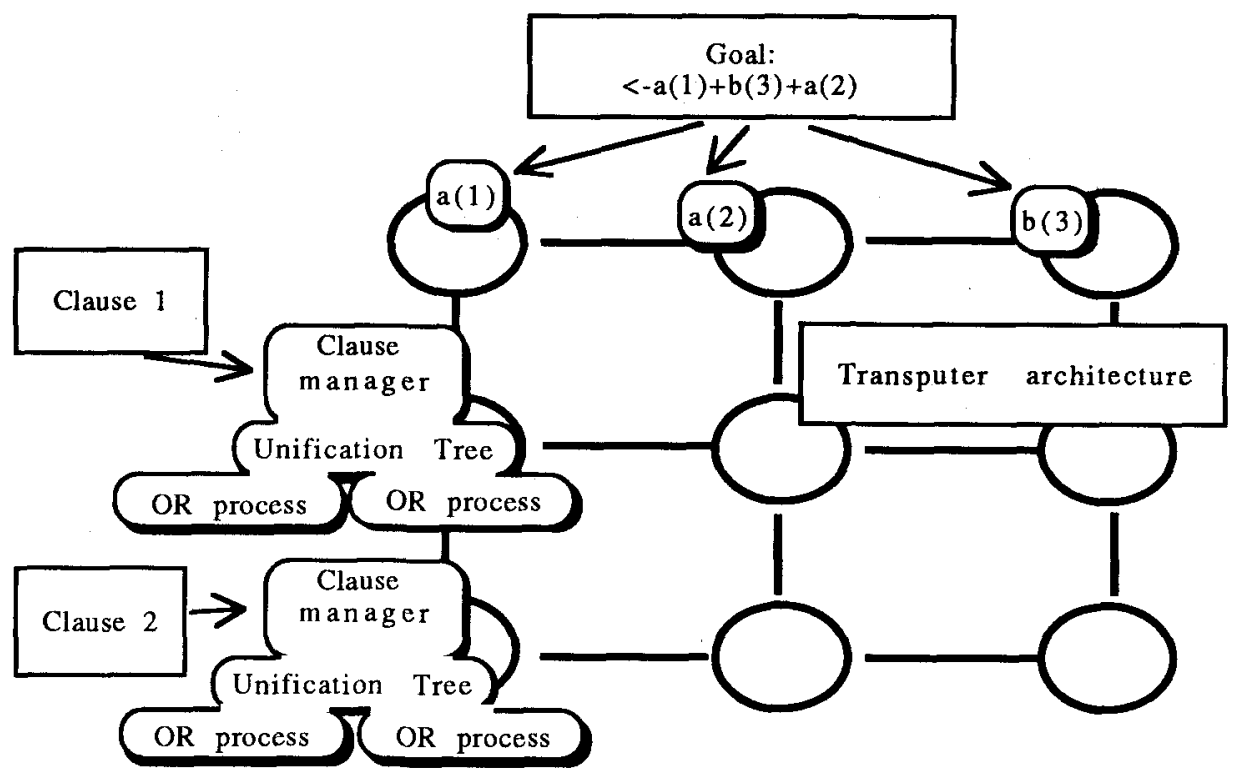

Fig. 3. Clause managers distribution.

3 - Distribution of clause managers and OR Processes: intra-method parallelism In this strategy of allocation, as in the previous case, all clause managers are distributed on distinct nodes. Moreover the leaves of each unification tree (i.e., the OR processes) are spawned on different nodes.

In this way, both the execution of different clauses and the guard evaluation of same clause activations are truly concurrent.

A finer degree of parallelism for objects is achieved: as in the previous case different requests can be served in parallel. In addition, more than one request of the same method can be served in a truly parallel way; also the guard evaluation of more than one invocation of the same method by a single instance is truly concurrent. No parallelism in accepting the same method request is possible.

4 - Distribution of clause managers and unification trees: a higher degree of intramethod parallelism

This allocation policy is the most distributed one, because even the unification processes (i.e., the nodes of the unification trees) are mapped on distinct nodes (see figure 4). In this way, also different applications of the same clause are executed in true concurrency.

From the object-oriented point of view this policy increases the degree of intramethod parallelism, if compared with the policy described in 3: the acceptance of a method request is implemented by several, truly parallel processes. 


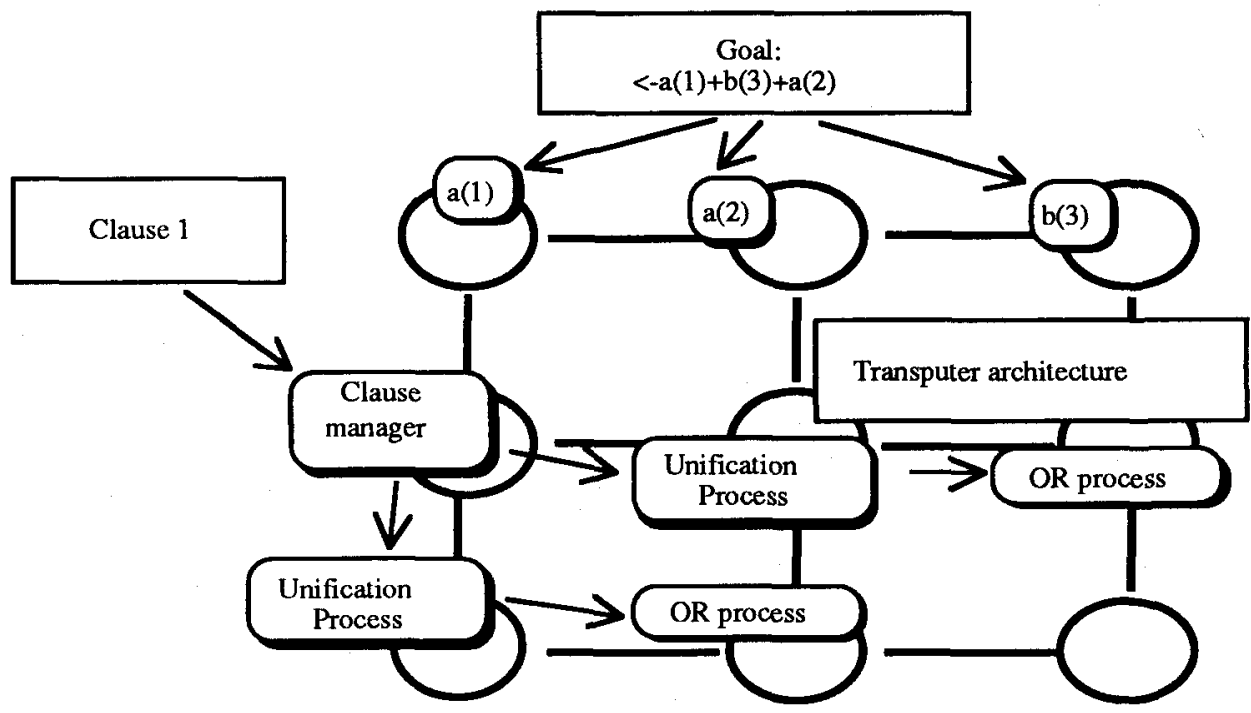

Fig. 4. The distribution of clause managers and unification trees.

\section{Experimental Results}

In the following, we present some experimental results obtained by the application of the policies described above to the following program P1:

$$
\begin{array}{ll}
\text { goal: } & \leftarrow \text { b }+a+c+d+a+d \\
\text { c1: } a+d & \leftarrow \text { truel } f+c \\
\text { c2: } f+e & \leftarrow \text { truel } \\
\text { c3: } \quad b+c & \leftarrow \text { truel } f+c
\end{array}
$$

The AND processes "b", "a", "c", "d", "a", "d", corresponding to the initial goal, run in parallel. The goal $\leftarrow \mathrm{b}+\mathrm{a}+\mathrm{c}+\mathrm{d}+\mathrm{a}+\mathrm{d}$ can be reduced by a single application of clause c3 and by two applications of clause $\mathrm{c} 1$.

The simulation of a non-propositional program is performed by introducing three parameters $\mathrm{Tu}, \mathrm{Tg}, \mathrm{Tb}$, which implicitly characterise the complexity of the logic program:

- Tu represents the load due to the unification of a single head, which impacts on the acceptance time of a method;

- $\mathrm{Tg}$ is the time due to the guard evaluation, and it influences the acceptance time of a method;

- Tb simulates the execution time of the body, that is the execution time of a method.

Experimental results are obtained by applying the above policies to different load conditions (achieved by varying $\mathrm{Tu}, \mathrm{Tg}, \mathrm{Tb}$ ).

The chosen parameters do not consider the time spent for the message transmission. In the non-propositional case the size of exchanged messages increases because of variable bindings carried in messages, thus influencing the choice of the optimal allocation policy. Nevertheless, in the considered Transputer architecture, an 
important part of the time taken to exchange messages is due to the initial phase, to define the connection between the two communicating processes. Thus, also a propositional simulation that considers messages of fixed size can give significant information about the behaviour of the non propositional case. In addition, on the one hand, while we have not considered the physical transmission time of messages of different size, a unification time ( $\mathrm{Tu}$ ) simulate the computational load required to the receipt of a unification message. On the other hand, the simulation parameter $\mathrm{Tb}$ includes the time taken to prepare message to send. Further detail about a complete distributed unification can be found in [8].

The analysis of timing results (see figures $5,6,7$ ) shows that the choice of the optimal allocation policy is influenced by the complexity of the program to be executed, given a target architecture.

More distributed policies produce an overhead due both to communication and to remote processes creation, whereas their parallelism allow a better exploitation of physical resources. In figure 5 execution times for the program P1 are presented.

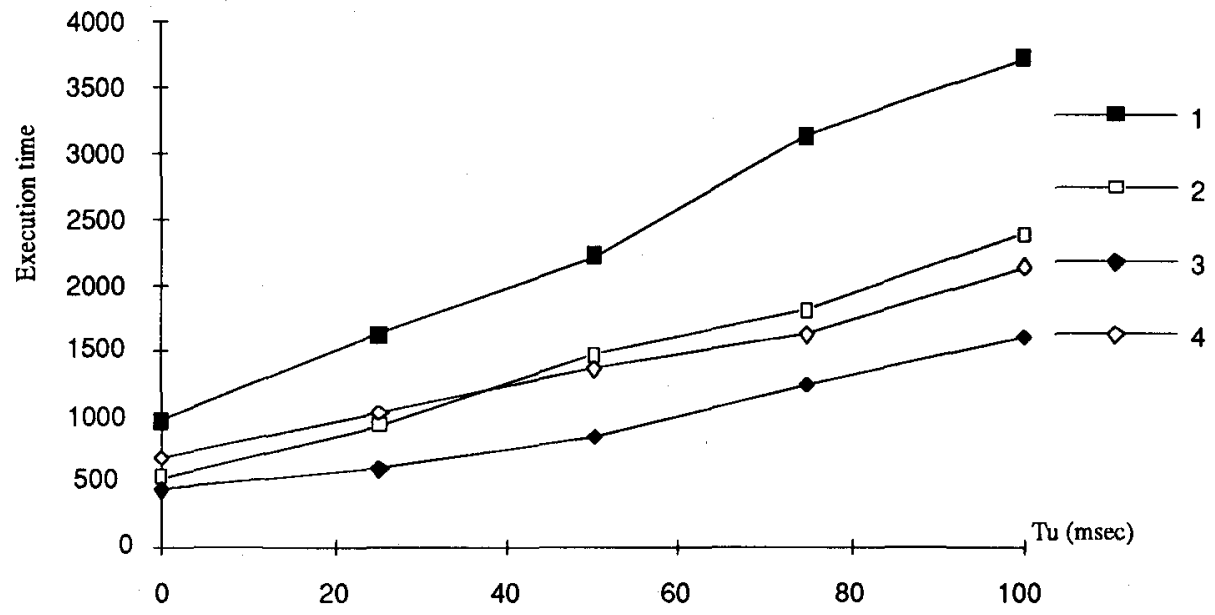

Fig 5. Execution Times (msec) obtained by varying the unification load $\mathrm{T}_{\mathrm{u}}$.

Each time value is obtained by assigning a particular value to $\mathrm{Tu}$, ranging from 0 to $150 \mathrm{~ms}$.

It clearly emerges from the diagram 5 that the best allocation policy is policy 3 . In fact, for the particular architectural configuration, this policy provides the best trade-off between the overhead due to distribution (remote processes creation and communication load) and the exploitation of available resources.

We could also notice that this policy obtains better results than the more distributed one (4) even with heavy unification loads. This can be explained with the limited number of available processors (16) which seems inadequate to the total number of spawned processes. Tests on programs with a lower number of generated processes confirm this way of interpretation.

As expected, the single node allocation policy produces the worst results. 
Focusing on policy 2 and 4 , we should notice that the less distributed one (2) gives better results than the more distributed one (4) for low unification times, and worse results for higher unification loads. In particular we have found a threshold for a unification time of about $30 \mathrm{~ms}$.

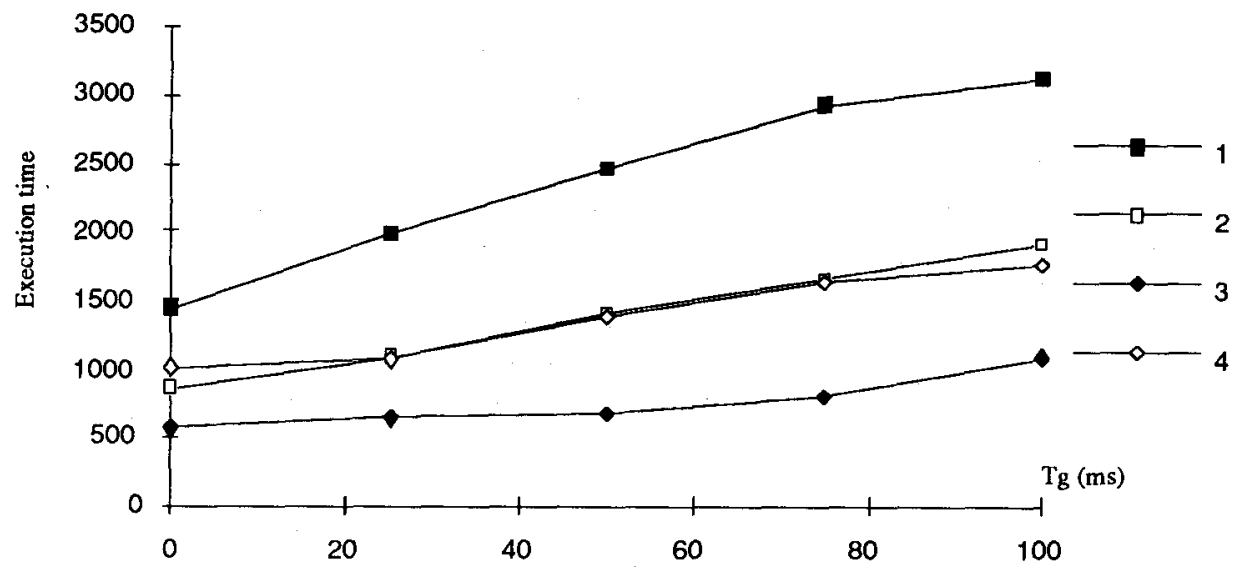

Fig. 6. Execution Times (msec) obtained by varying the guard evaluation load $\mathrm{T}_{\mathrm{g}}$.

Figures 6 and 7 show performance curves obtained for the example P1 when varying the parameters $T_{g}$ and $T_{b}$ respectively. These tests adopt a fixed unification load $(\mathrm{Tu}=15 \mathrm{~ms})$. The Tu value has been obtained by estimating the average cost of a single head unification on a Transputer architecture (for details, see [8]).

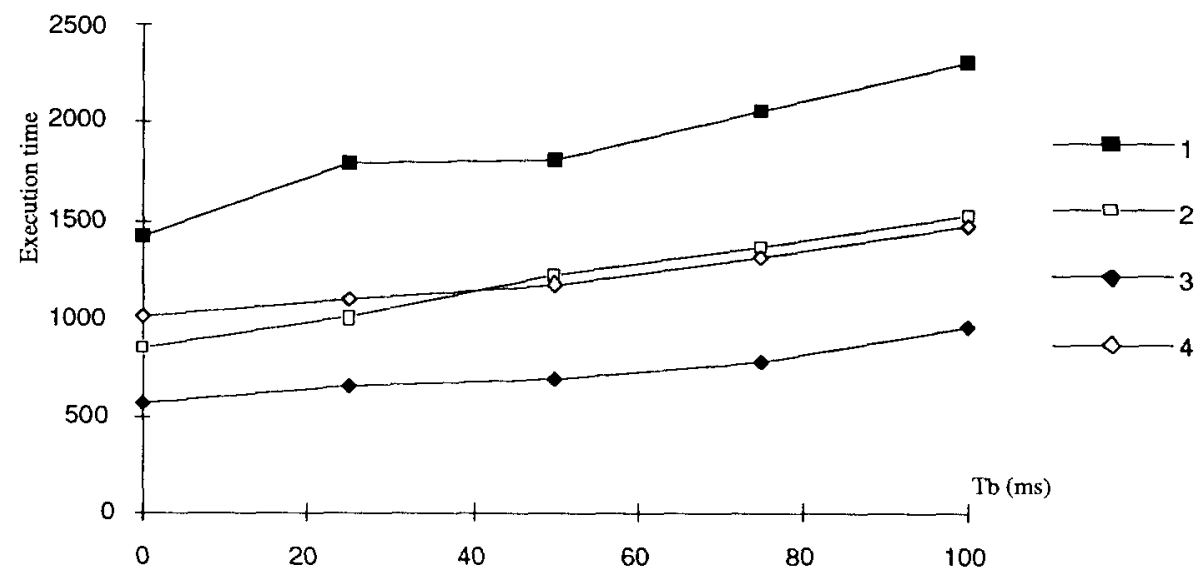

Fig. 7. Execution Times obtained by varying the body load $\mathrm{T}_{\mathrm{b}}(\mathrm{Tu}=15 \mathrm{~ms})$.

Experimental results obtained varying $\mathrm{Tg}$ (fig. 6) and $\mathrm{Tb}$ (fig. 7) show a similar behaviour in both cases. 
Comparing figure 5 with results in figures 6-7, execution times are lower in the first case than in the second one, due to the additional load introduced in tests 6-7.

Moreover, for the given architectural configuration, best execution times in figures 6 and 7 are obtained (as in figure 5) with policy 3. Also in these cases, load values (either $\mathrm{Tg}$ or $\mathrm{Tb}$ ) greater than a threshold make convenient to choose a more distributed policy (4) rather than a less distributed one (2).

As can be inferred from the figures, allocation policy 3 seems to give best results. In this policy, in fact, manager processes are allocated on separate nodes, whenever possible, together with all the unification processes of the tree. There is, in fact, a high coupling degree between processes in the same unification tree, while the computational load of each unification process is light. OR processes, however, are allocated on separate nodes since they carry on the greater part of the computation that consists in the guard evaluation and the body code execution.

However, if availability of physical nodes is not a problem (and this is not our case where only 16 nodes can be used), the allocation of manager and unification processes can take place on different nodes, in order to exploit completely the high parallelism degree of the incremental unification mechanisms (allocation policy 4).

\section{Conclusions and Future Works}

We have described some practical results in implementing a distributed logic language on a Transputer-based architecture. The language (called ROSE) is a multi-head clause language with restricted AND and OR-parallelism and can be suitably used for implementing parallel and distributed logic objects.

The results have been obtained (using an implementation supporting only the propositional case) by considering different allocation policies with an increasing degree of real parallelism, and simulating the computation times due to unification and guard and body evaluation.

This allows us to determine the best allocation policy for a distributed logic program, on the basis of an estimate of these computation times.

Of course, the more distributed allocation policies become convenient when computation times for unification, body and guard evaluation increase.

We are working on implementing the overall ROSE language taking into account also the distributed unification in the case of variables, the sequential part of ROSE and its integration with the parallel one. In this way, also logic objects with mutable state will be supported.

With reference to the distributed unification, we can take advantage of the implementation described in [8]. In that paper, we present the distributed implementation of a simple parallel logic language with OR-parallelism and focus on how the unification is performed in a distributed environment. The main problem we face is how and when to access remote data structures to perform the distributed unification, in order to reduce the amount of the inter-node communication.

In the future, we plan to execute more significant benchmark tests in order to better evaluate the implementation of ROSE and investigate more sophisticated allocation policies.

Moreover, we intend to compare practically our implementation of ROSE with that of the language STRAND88 [20], a STREAM-based language which is 
implemented on the Meiko Computer Surface. The goal is to show that, thanks to the lack of variables shared by AND processes, ROSE and logic objects realised on top of it are more suitable for a distributed implementation with respect to the case of STREAM-parallel languages.

\section{Acknowledgements}

This work has been partially supported by the M.U.R.S.T. $40 \%$ Project and by the C.N.R. "Progetto Finalizzato Sistemi Informatici e Calcolo Parallelo" (grant n. 92.01606.PF69). We thanks Antonio Brogi for the useful discussions about the ROSE language.

\section{References}

1. J.M. Andreoli, R. Pareschi: Linear objects: logical processes with built-in inheritance, Proc. 7th Int.1 Conf. on Logic programming ICLP'90, Jerusalem, (D.H.D. Warren and Peter Szeredi eds), The MIT Press, pp. 495510 (1990).

2. G. Agha: Actors: A Model of Concurrent Computation in Distributed Systems, The MIT Press, 1986.

3. A. Brogi: AND-Parallelism without Shared Variables, in Proc. Seventh International Conference on Logic Programming, Jerusalem, (D.H.D. Warren and Peter Szeredi Eds.), pp. 306-324, The MIT Press (1990).

4. A.Brogi, A.Ciampolini, E.Lamma, P.Mello: A Distributed Implementation for Parallel Logic Programming, in Proc. COMPEURO91, pp. 118-122, IEEE Computer Soc. Press (1991).

5. A.Brogi, A.Ciampolini, E.Lamma, P.Mello: The Implementation of a Distributed Model for Logic Programming based on Multiple-Headed Clauses, Information Processing Letters, Vol. 42, pages 331-338, Elsevier Science Publisher B.V.-North-Holland (1992).

6. A. Burns, Programming in occam-2, Addison-Wesley (1988).

7. J.S. Conery, D.F.Kibler: AND parallelism and non-determinism in logic programs, in New Generation Computing, Vol.3, pp. 43-70 (1985).

8. A.Ciampolini, E.Lamma, P.Mello, C.Stefanelli, Implementing Unification in a Distributed Environment, Proc. AICA-93, International Section, Gallipoli (Lecce), September 1993.

9. J.S: Conery: Logical Objects, Proc. 5th Int.1 Conf. on Logic programming ICLP'88, Seattle, (R.A. Kowalski and K.A. Bowen eds), The MIT Press, pp. 420-434 (1988).

10. D. DeGroot et al. editors, Distributed and Parallel Implementations of Logic Programming Systems, Proc. of the JICSLP92 Post-Conf. Workshop.

11. M. Falaschi, G. Levi, C. Palamidessi: A Synchronization Logic: Axiomatic and Formal Semantics of Generalized Horn Clauses, Information and Control, 60, 1-3, pp. 36-69 (1984). 
12. I. Foster: Parallel Implementation of Parlog, in Proceedings of the 1988 International Conference on Parallel Processing, V.2, pp. 9-16, 1998.

13. INMOS, Transputer Reference Manual, Prentice Hall (1988).

14. K.Kahn, E.D.Tribble, M.S.Miller, D.G.Bobrow: Objects in Concurrent Logic Programming Languages, OOPSLA'86, Portland, Oregon, September 1986.

15. MEIKO, CSTools: A Technical Overview, Meiko Technical Report S020512S (1989).

16. L. Monteiro: A proposal for distributed programming in logic, in Implementations of Prolog (J.A. Campbell ed.), pp. 329-340, Ellis Horwood (1984).

17. L. Monteiro: Distributed Logic: A Theory of Distributed Programming in Logic, Technical Report, Universidade Nova de Lisboa, (1986).

18. K.Nakajima et al.: Distributed Implementation of KL1 on the Multi-PSI/V2, Proc. 6th International Conference on Logic Programming, Lisbon (P), (G. Levi, M. Martelli eds.), pp. 436-451, The MIT Press (1989).

19. E. Y. Shapiro: The Family of Concurrent Logic Programming Languages, ACM Computing Surveys, 21:3, pp.412-510 (1989).

20. I.Foster, S. Taylor: STRAND: New Concepts in ParallelProgramming, Prentice Hall, 1990.

21. K.Ueda, M.Morita: A New Implementation Technique for Flat GHC, Proc. Seventh International Conference on Logic Programming, Jerusalem, (D.H.D. Warren, Peter Szeredi eds.), pp. 3-17, The MIT Press (1990).

22. D.H.D. Warren: An Abstract Prolog Instruction Set, SRI Technical Note 309, SRI International (1983). 